\title{
Heterozygous CTLA-4 splice site mutation c.458-1G>C presenting with variable degree of immune dysregulation and autoimmunity in three generation kindred of Caribbean descent
}

\author{
UPENDRA MAHAT ${ }^{1}$, Nila Ambani ${ }^{1}$, seth rotz $^{1}$, and Kadakkal Radhakrishnan ${ }^{1}$ \\ ${ }^{1}$ Cleveland Clinic
}

October 21, 2020

\begin{abstract}
Cytotoxic T-lymphocyte-associated protein 4 (CTLA-4) is an immune checkpoint, which downregulates T cell activation and $\mathrm{T}$ regulatory cell function. CTLA-4 haploinsufficiency (CTLA4 HI) leads to T cell hyperactivation, immune dysregulation, lymphoproliferation and cancer predisposition. Less well understood is the penetrance and expressivity of CTLA-4 mutations. We describe five members of a single family with heterozygous CTLA-4 splice site mutation c.458-1G>C, previously shown to result in CTLA-4 HI, who presented with variable degree of immune dysfunction, lymphocytic infiltration and autoimmunity. The host, environmental and the epigenetic factors affecting the penetrance and expressivity of CTLA-4 mutations merits further investigation.
\end{abstract}

\section{Introduction:}

CTLA-4 is an immunoglobulin superfamily receptor, primarily expressed by activated T cells and FOXP3 ${ }^{+}$ regulatory T cells. ${ }^{1}$ CTLA-4 down-regulates the $\mathrm{T}$ cell mediated immune response, and thus functions as an immune checkpoint. ${ }^{2}$ Homozygous CTLA-4 deficiency in mice causes severe fatal immune dysfunction..$^{3,4}$ In humans, CTLA-4 haploinsufficiency (HI) causes polyclonal $\mathrm{T}$ cell hyperactivation, and present with immunodeficiency, antibody mediated autoimmunity and lymphoproliferation in non-lymphoid organs such as gut, lungs, CNS, bone marrow, etc. ${ }^{5-8}$ Furthermore, CTLA-4 HI predisposes patients to development of various malignancies such as lymphoma and gastric cancers. ${ }^{9,10}$

\section{Method:}

We describe the incompletely penetrant and variably expressed clinical phenotype of a patient of Caribbean descent with CTLA-4 HI, and his 4 related family members (Family pedigree, clinical presentation and genetic defect as depicted in Figure 1A-C). The study was approved by the institutional IRB. The informed consents were obtained as per the IRB guidelines.

\section{Results:}

The index patient III.1 (previously published ${ }^{11}$ ) is 24-year-old male, who was diagnosed with EBV positive classical Hodgkin lymphoma (cHL) of nodular sclerosis histology at 11 year of age. He achieved complete remission with multiagent chemotherapy and radiation. Subsequently, he developed autoimmune hemolytic anemia (AIHA), and then immune thrombocytopenia (ITP). At 17 years of age, he was diagnosed with autoimmune lymphoproliferative syndrome (ALPS), and further genetic testing revealed heterozygous CTLA-4 splice mutation c.458-1G $>$ C. Four years later, the patient developed autoimmune lymphocytic enterocolitis (ALE). A year later, at 22 years of age, he was diagnosed with a new EBV+ cHL of mixed cellularity subtype. He was treated with further courses of chemotherapy, and complete remission was achieved. Further, 
the patient underwent allogeneic peripheral stem cell transplantation from matched unrelated donor. At the present time, he remains in complete remission from both cHL, and autoimmune conditions.

Patient III.3 is 14 years old male, and is the maternal cousin brother of the proband. He developed chronic progressive diarrhea since he was 1 year old. At the age of 5 years, he developed multiple recurrent anal warts. Esophagogastroduodenoscopy (EGD) and colonoscopy revealed ALE. He was started on oral tacrolimus, which improved his symptoms. He was treated with multiple courses of corticosteroids. Few months later, he developed right upper quadrant abdominal pain. Imaging studies demonstrated multiple small gallbladder calculi and choledocholithiasis, requiring ERCP and removal of CBD stones. A trial of sirolimus worsened his symptoms and he had to be restarted back on tacrolimus with improvement in his gastrointestinal symptoms. His genetic testing revealed the same CTLA- 4 spice site mutation as the proband. Since the age of 12 years, he was started on abatacept, a fusion protein composed of Fc region of the IgG1 fused to the extracellular domain of CTLA-4, every 4 weeks, along with low dose tacrolimus and sirolimus with good improvement of symptoms. He has developed multiple nutritional deficiencies such as copper, vitamin A, and vitamin D, which improved with supplementation. Of note, patient also developed chronic EBV viremia since the age of 10 years. However, no signs of lymphoma or lymphoproliferative disorders have been identified.

Patient III.4 is the paternal cousin sister of the proband, who is 20 years old. She developed ITP at 9 years of age. When she was 12 years old, she was admitted with chronic diarrhea and excessive weight loss, prompting EGD and colonoscopy, which revealed ALE. The genetic testing confirmed CTLA4 c.458-1 G>C mutation. She was started on oral tacrolimus since the age of 14 years, with near complete resolution of GI symptoms associated with ALE. Of note, she was also found to have EBV viremia since the age of 16 years. However, she has not developed lymphoproliferative disorders or lymphoma.

Patient II.3 is the maternal uncle of the proband, and the father of patient III.3 and III.4. He is 43 years old and was diagnosed with ALE at 28 years of age. Later, he was found to have chronic hypogammaglobinemia and was clinically diagnosed with common variable immunodeficiency (CVID). He developed AIHA at the age of 29 years. When he was 32 years old, he was diagnosed with atypical marginal zone non-Hodgkin lymphoma with CNS metastasis. No genetic testing has been performed.

Patient II.5 is the maternal uncle of the proband, who had history of cataract at the age of 6 years. He also had history of multiple surgeries for glaucoma in his childhood. He was diagnosed with juvenile rheumatoid arthritis at the age of 9 months. Later, he developed ITP, AIHA, ALE, hypothyroidism, hypogonadism and insulin dependent DM.

\section{Conclusion and discussion:}

The proband and the family members in our cohort harbors CTLA-4 splice site mutation, c. $458-1 \mathrm{G}>\mathrm{C}$, in the region of intron 2. As described above, the patients presented with variable degree of immune dysregulation and autoimmunity. The mutation has been previously shown to disrupt the acceptor site of second CTLA-4 intron, and cause defective transcription of third exon. ${ }^{12}$ The common manifestations in the majority of the patients described by previous reports have lymphoproliferation, lymphocytic infiltration of non-lymphoid organs, autoimmune cytopenias and hypogammaglobinemia. ${ }^{8,12}$ Mutations described in total of 18 patients in those reports spanned across the CTLA-4 gene, including non-sense mutations such as c.151C $>$ t (p.R51X), c.105C >A (p.C35*), c.208C > T (p.R70W), c.371A >C (p.T124P), c.223C > T (p.R75W) and c.2T $>$ C (p.?), splice site mutations such as c110 $+1 \mathrm{G}>\mathrm{T}$ in intron 1 , c.458-1G $>\mathrm{C}$ in intron 2, c.567+5G $>\mathrm{C}$ in intron 3, and frameshift deletion c.75delG> p.L28Fs* $44 .^{8,12}$ Although the nature and the location of these mutations were heterogeneous, all of these mutations led to decreased CTLA-4 expression in Treg cells compared to healthy controls. Furthermore, the reduction was more pronounced on T cell activation for unclear reason. The Treg cells' CTLA-4 dependent suppressive function, ligand binding and transendocytosis of CD80 were uniformly impaired.

One patient reported by Kuehn et al harbored c.458-1G $>$ C mutation, as in our cohort, and presented with diffuse lymphadenopathy, splenomegaly, AIHA, ITP, ALE and childhood onset EBV positive HL. ${ }^{12}$ The severity and expressivity of immune dysregulation in our cohort appears somewhat more penetrant in male 
gender. Gastrointestinal manifestations especially ALE appear more common and may pose management challenges. The role of abatacept in the management of these patients may need to be studied further, alone or in conjunction with tacrolimus and/or sirolimus. Two patients in our cohort presented with lymphoma (two episodes of EBV driven cHL of different histologies in one patient and one case of atypical marginal zone non-Hodgkin lymphoma). An earlier published worldwide cohort of 184 patients with CTLA-4 mutations, has reported various malignancies in these patients, such as lymphoma, gastric cancers, multiple myeloma and metastatic melanoma. ${ }^{9}$

In conclusion, heterozygous CTLA-4 mutation and the associated immune dysregulation is a complex entity which is both phenotypically as well as genotypically heterogeneous. Although the previous studies have extensively investigated the essential role of CTLA-4 in T and B cell homeostasis, our understanding of the molecular, genetic and epigenetic regulation of its penetrance and expressivity remains limited, and merits further investigation. Defining the genotypic and phenotypic correlates may help refine the management options for this intriguing disorder.

\section{Conflict of interest:}

Authors have no conflict of interest to disclose.

\section{Consent:}

Appropriate consents were obtained from the participants as per the IRB guidelines.

\section{Acknowledgements:}

We would like to sincerely acknowledge the participants of this study.

\section{References:}

1. Buchbinder EI, Desai A. CTLA-4 and PD-1 Pathways: Similarities, Differences, and Implications of Their Inhibition. Am J Clin Oncol. 2016;39(1):98-106.

2. Syn NL, Teng MWL, Mok TSK, Soo RA. De-novo and acquired resistance to immune checkpoint targeting. Lancet Oncol. 2017;18(12):e731-e741.

3. Tivol EA, Borriello F, Schweitzer AN, Lynch WP, Bluestone JA, Sharpe AH. Loss of CTLA-4 leads to massive lymphoproliferation and fatal multiorgan tissue destruction, revealing a critical negative regulatory role of CTLA-4. Immunity. 1995;3(5):541-547.

4. Waterhouse P, Penninger JM, Timms E, et al. Lymphoproliferative disorders with early lethality in mice deficient in Ctla-4.Science. 1995;270(5238):985-988.

5. Hudson LL, Rocca K, Song YW, Pandey JP. CTLA-4 gene polymorphisms in systemic lupus erythematosus: a highly significant association with a determinant in the promoter region. Hum Genet.2002;111(45):452-455.

6. Kouki T, Sawai Y, Gardine CA, Fisfalen ME, Alegre ML, DeGroot LJ. CTLA-4 gene polymorphism at position 49 in exon 1 reduces the inhibitory function of CTLA- 4 and contributes to the pathogenesis of Graves' disease. J Immunol. 2000;165(11):6606-6611.

7. Oaks MK, Hallett KM. Cutting edge: a soluble form of CTLA-4 in patients with autoimmune thyroid disease. J Immunol.2000;164(10):5015-5018.

8. Schubert D, Bode C, Kenefeck R, et al. Autosomal dominant immune dysregulation syndrome in humans with CTLA4 mutations. Nat Med.2014;20(12):1410-1416.

9. Egg D, Schwab C, Gabrysch A, et al. Increased Risk for Malignancies in 131 Affected CTLA4 Mutation Carriers. Front Immunol.2018;9:2012. 
10. Schwab C, Gabrysch A, Olbrich P, et al. Phenotype, penetrance, and treatment of 133 cytotoxic T-lymphocyte antigen 4-insufficient subjects.J Allergy Clin Immunol. 2018;142(6):1932-1946.

11. Mahat U, Terzioglu MK, Buhtoiarov I. CTLA4 haploinsufficiency as a predisposition to classical Hodgkin lymphoma. Pediatr Hematol Oncol. 2020;37(2):176-183.

12. Kuehn HS, Ouyang W, Lo B, et al. Immune dysregulation in human subjects with heterozygous germline mutations in CTLA4. Science.2014;345(6204):1623-1627.

\section{Legends:}

Figure 1: Figure illustrating A. Family pedigree with members from three generation family with heterozygous CTLA-4 mutation and variable clinical phenotypic expression, B. Clinical presentation of the members of the family depicting key immune manifestations and C. CTLA-4 mutation location and protein structure showing key domains.

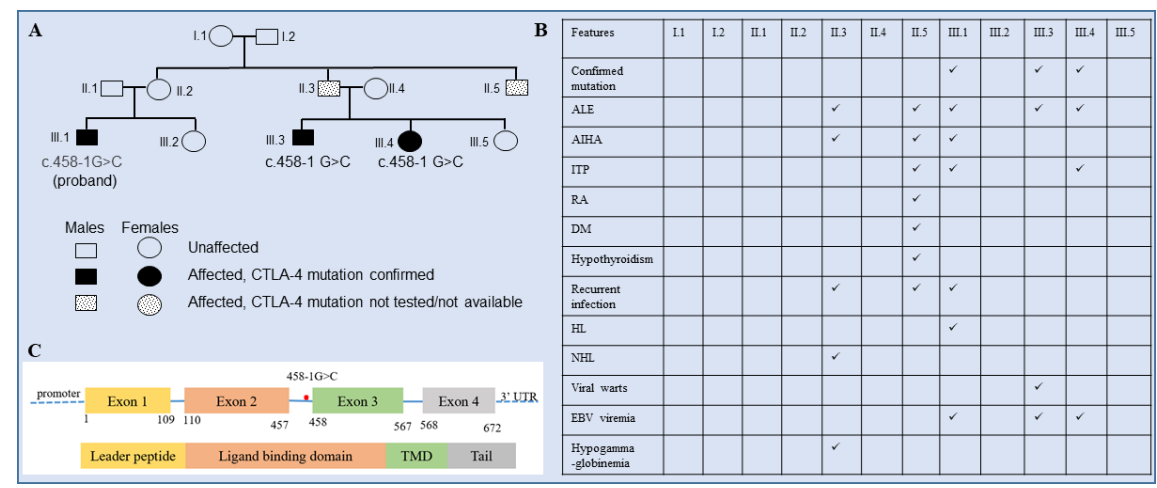

